\title{
Minmax regret approach and optimality evaluation in combinatorial optimization problems with interval and fuzzy weights
}

\author{
Adam Kasperski \\ Institute of Industrial Engineering and Management, Wroctaw University of Technology, \\ Wybrzeże Wyspiańskiego 27, 50-370 Wroctaw, Poland, adam.kasperski@pwr.wroc.pl \\ Paweł Zieliński* \\ Institute of Mathematics and Computer Science Wroctaw University of Technology, \\ Wybrzėze Wyspiańskiego 27, 50-370 Wroctaw, Poland, pawel.zielinski@pwr.wroc.pl
}

\begin{abstract}
This paper deals with a general combinatorial optimization problem in which closed intervals and fuzzy intervals model uncertain element weights. The notion of a deviation interval is introduced, which allows us to characterize the optimality and the robustness of solutions and elements. The problem of computing deviation intervals is addressed and some new complexity results in this field are provided. Possibility theory is then applied to generalize a deviation interval and a solution concept to fuzzy ones.
\end{abstract}

Keywords: Minmax regret; Interval; Possibility theory; Combinatorial optimization

\section{Introduction}

A wide class of deterministic combinatorial optimization problems with a linear objective function consists in finding a feasible solution from a finite set whose total weight is maximal or minimal. Typically, a set of feasible solutions is formed by subsets of a given finite set of elements $E$. Every element in $E$ has a nonnegative weight and we seek a feasible solution whose total weight is minimal or maximal. An element $e \in E$ is called optimal if it is a part of an optimal solution.

In many real-world applications the element weights may be ill-known or uncertain. The simplest form of the uncertainty representation is to specify the element weights as

*Corresponding author 
closed intervals. Every precise instantiation of the weights is called a configuration (it is also called a scenario in the literature). Now a solution (an element) is possibly optimal if it is optimal for at least one configuration (the event that it will be optimal is possible). Similarly, a solution (an element) is necessarily optimal if it is optimal for all configurations (the event that it will be optimal is sure). The notions of possible and necessary optimality of solutions and elements have been already introduced in the literature for some particular problems: in $[8,9,10,12,14]$ for scheduling problems, in [21] for matroidal problems, in $[16,17,18]$ for linear programming, in [19] for shortest path and in [27] for minimum spanning tree. In this paper, we generalize the optimality notions to all combinatorial optimization problems with interval weights. We show that both possible and necessary optimality can be expressed by means of the so-called deviation interval, which provides some additional information under uncertainty. The upper bound of the deviation interval of a solution is called in the literature a maximal regret and it is a natural criterion for choosing a solution under the interval representation of uncertainty (see [22]). Namely, in the minmax regret approach we seek a solution that minimizes the maximal regret and this approach to combinatorial optimization has been extensively studied in the recent literature (see, e.g. [2, 3, 5, 6, 19, 20, 24, 27] and [4] for a recent survey).

In this paper, we show some general relationships between the deviation interval, the possible and necessary optimality and the minmax regret approach. We provide some new results and we generalize the results known for some particular problems. We also discuss the problem of computing bounds of the deviation interval for a given solution or an element. In particular, we provide new complexity results for some basic problems such as shortest path, minimum assignment and minimum $s-t$ cut. The results obtained for the interval-valued case can be generalized so that uncertainty is modeled in a more sophisticated manner. The key idea is to generalize the classical closed interval to a fuzzy one. A fuzzy interval is regarded as a possibility distribution describing the set of more or less plausible values of an element weight. Using possibility theory [11] we can generalize the notion of the deviation interval to the fuzzy case. From a fuzzy deviation interval, that is a possibility distribution representing a set of plausible values of solution (element) deviations, we can derive the degrees of possible and necessary optimality of a solution (an element). As in the interval case, we can use an upper bound of the fuzzy deviation interval to choose a solution. This leads to the concept of a necessary soft optimality, which has been originally proposed in $[17,18]$ for linear programming problem with a fuzzy objective function. Choosing a best necessarily soft optimal solution is a direct generalization of the minmax regret approach to the fuzzy case.

This paper is organized as follows. In Section 2 we recall a formulation of the deterministic combinatorial optimization problem. In Section 3 we discuss the problem with uncertain weights modeled as closed intervals. We introduce the concept of the deviation interval and we show some various properties of this notion. In particular, we show that computing the lower bound of the deviation interval for a given element is NP-hard for some basic problems. Section 4 is devoted to a general combinatorial optimization problems with uncertain weights modeled by fuzzy intervals. We show how the notions and results presented in Section 3 can be naturally generalized to the fuzzy case. 


\section{Deterministic combinatorial optimization problem}

Let $E=\left\{e_{1}, \ldots, e_{n}\right\}$ be a finite set of elements and let $\Phi \subseteq 2^{E}$ be a set of subsets of $E$ called the set of feasible solutions. A nonnegative real weight $w_{e}$ is given for every element $e \in E$. A combinatorial optimization problem $\mathcal{P}$ with a linear objective function consists in finding a feasible solution $X \in \Phi$ whose total weight $\sum_{e \in X} w_{e}$ is minimal. This formulation encompasses a wide class of problems such as ShorTest Path, Minimum Spanning Tree, Minimum Assignment and Minimum s-T Cut.

We call an element $e \in E$ optimal if it is a part of an optimal solution to problem $\mathcal{P}$. If a solution (an element) is not optimal a natural question arises how far from optimality this solution (element) is. To answer this question one can introduce the concept of a deviation. A deviation of solution $X \in \Phi$ is defined in the following way:

$$
\delta_{X}=\sum_{e \in X} w_{e}-\min _{Y \in \Phi} \sum_{e \in Y} w_{e}
$$

Similarly, a deviation of element $f \in E$ is defined as follows:

$$
\delta_{f}=\min _{Y \in \Phi_{f}} \sum_{e \in Y} w_{e}-\min _{Y \in \Phi} \sum_{e \in Y} w_{e}
$$

where $\Phi_{f}$ is the set of all feasible solutions that contain element $f$. It is clear that solution $X$ (element $f)$ is optimal if and only if $\delta_{X}=0\left(\delta_{f}=0\right)$.

\section{Interval combinatorial optimization problem}

Assume that the values of the weights associated with the elements are only known to belong to the intervals $W_{e}=\left[\underline{w}_{e}, \bar{w}_{e}\right], \underline{w}_{e} \geq 0$. If the weight of $e$ is precisely known, then it is represented by a degenerate interval $\left[\underline{w}_{e}, \bar{w}_{e}\right]$ such that $\underline{w}_{e}=\bar{w}_{e}$. We assume that the element weights are unrelated to one another. A vector $\boldsymbol{w}=\left(w_{e}\right)_{e \in E}, w_{e} \in W_{e}$, that represents an assignment of weights $w_{e}$ to elements $e \in E$ is called a configuration (it is also called a scenario in the literature). Thus every configuration expresses a realization of the weights that may occur with a positive but perhaps unknown probability. We denote by $\Gamma$ the set of all the configurations, i.e. $\Gamma=\times_{e \in E}\left[\underline{w}_{e}, \bar{w}_{e}\right]$. For a given solution $X \in \Phi$, we define its weight under a fixed configuration $\boldsymbol{w} \in \Gamma$ as $F(X, \boldsymbol{w})$. We will denote by $F^{*}(\boldsymbol{w})$ the value of the weight of an optimal solution under configuration $\boldsymbol{w} \in \Gamma$, that is $F^{*}(\boldsymbol{w})=\min _{X \in \Phi} F(X, \boldsymbol{w})$.

Now the optimality of solutions and elements depends on configuration $\boldsymbol{w} \in \Gamma$ and it can be characterized in the following way. A solution $X \in \Phi$ (an element $e \in E$ ) is said to be possibly optimal if there exists a weight configuration $w \in \Gamma$ for which it is optimal. A solution $X \in \Phi$ (an element $e \in E$ ) is said to be necessarily optimal if it is optimal for all weight configurations $\boldsymbol{w} \in \Gamma$. A possibly (necessarily) optimal solution is composed of possibly (necessarily) optimal elements. However, the converse statement is not true, since there may exist a non-possibly (non-necessarily) optimal solution entirely composed 
of possibly (necessarily) optimal elements. Furthermore, a necessarily optimal solution may not exist, even if there are some necessarily optimal elements [21].

We can express both possible and necessary optimality using the concept of deviation. Let $\delta_{X}(\boldsymbol{w})=F(X, \boldsymbol{w})-F^{*}(\boldsymbol{w})$ denote the deviation of solution $X$ under a specified configuration $\boldsymbol{w} \in \Gamma$. We can now compute $\underline{\delta}_{X}=\min _{\boldsymbol{w} \in \Gamma} \delta_{X}(\boldsymbol{w})$ and $\bar{\delta}_{X}=\max _{\boldsymbol{w} \in \Gamma} \delta_{X}(\boldsymbol{w})$, that is the smallest and the largest deviation for solution $X$ over set $\Gamma$. They determine a solution deviation interval $\Delta_{X}=\left[\underline{\delta}_{X}, \bar{\delta}_{X}\right]$. The upper bound $\bar{\delta}_{X}$ is called in the literature the maximal regret of $X$ and configuration $\boldsymbol{w} \in \Gamma$ that maximizes deviation $\delta_{X}(\boldsymbol{w})$ is called a worst case configuration for $X$. In a similar way we define the element deviation $\delta_{f}(\boldsymbol{w})=\min _{Y \in \Phi_{f}} F(Y, \boldsymbol{w})-F^{*}(\boldsymbol{w})$ under $\boldsymbol{w} \in \Gamma$ and compute the quantities $\underline{\delta}_{f}$ and $\bar{\delta}_{f}$, which form an element deviation interval $\Delta_{f}=\left[\underline{\delta}_{f}, \bar{\delta}_{f}\right]$. It is easy to check that solution $X$ (element $f$ ) is possibly optimal if and only if $\underline{\delta}_{X}=0\left(\underline{\delta}_{f}=0\right)$ and solution $X$ (element $f)$ is necessarily optimal if and only if $\bar{\delta}_{X}=0\left(\bar{\delta}_{f}=0\right)$.

We now address the question of choosing a solution under interval weights. Our aim is to compute a solution that behaves reasonably well under any possible weight configuration $\boldsymbol{w} \in \Gamma$. Obviously, a necessarily optimal solution is an ideal choice because it is optimal regardless of weight realizations. Furthermore, if problem $\mathcal{P}$ is polynomially solvable, then detecting a necessarily optimal solution can be done in polynomial time as well using the results obtained in [20]. It is enough to compute an optimal solution $Y$ under weights $w_{e}=\frac{1}{2}\left(\underline{w}_{e}+\bar{w}_{e}\right)$ for all $e \in E$. If there is a necessarily optimal solution, then $Y$ must be necessarily optimal. However, this approach has a drawback. The necessary optimality is too strong a criterion for choosing a solution because a necessarily optimal solution rarely exists. Observe, that a necessarily optimal solution has the maximal regret equal to 0 . Therefore, it is reasonable to compute a solution whose maximal regret is minimal, minimizing in this way a distance to the necessary optimality. We thus consider problem $\min _{X \in \Phi} \bar{\delta}_{X}$, which belongs to the class of robust discrete optimization problems described in book [22]. This class has been extensively studied in the recent literature (see, e.g. $[2,3,5,6,19,20,24,27]$ and [4] for a recent survey).

\subsection{A characterization of optimal minmax regret solutions}

Among the configurations of $\Gamma$ a crucial role is played by the extreme ones, which belong to $\times_{e \in E}\left\{\underline{w}_{e}, \bar{w}_{e}\right\}$. Let $A \subseteq E$ be a fixed subset of elements. In configuration $\boldsymbol{w}_{A}^{+}$all elements $e \in A$ have weights $\bar{w}_{e}$ and all the remaining elements have weights $\underline{w}_{e}$. Similarly, in configuration $\boldsymbol{w}_{A}^{-}$all elements $e \in A$ have weights $\underline{w}_{e}$ and all the remaining elements have weights $\bar{w}_{e}$. It is easily seen that $\underline{\delta}_{X}=\delta_{X}\left(\boldsymbol{w}_{X}^{-}\right)$and $\bar{\delta}_{X}=\delta_{X}\left(\boldsymbol{w}_{X}^{+}\right)$. In consequence, $\boldsymbol{w}_{X}^{+}$is the worst case configuration for solution $X$. Furthermore, $X$ is possibly optimal if and only if it is optimal in configuration $\boldsymbol{w}_{X}^{-}$and it is necessarily optimal if and only if it is optimal in configuration $\boldsymbol{w}_{X}^{+}$. We can see now that if problem $\mathcal{P}$ is solvable in polynomial time, then the optimality of a given solution $X \in \Phi$ can be characterized in polynomial time and its maximal regret can be computed in polynomial time as well. It is worth pointing out that for the linear programing problem with interval coefficients in the objective function the 
problem of computing the maximal regret of a feasible solution $X$ turns out to be strongly NP-hard [7].

Contrary to solutions, the computation of $\underline{\delta}_{f}$ and $\bar{\delta}_{f}$ for a given element $f$ is far from being trivial even if problem $\mathcal{P}$ is polynomially solvable. It follows easily that $\delta_{f}(\boldsymbol{w})$ attains minimum (maximum) at an extreme configuration $\boldsymbol{w} \in \times_{e \in E}\left\{\underline{w}_{e}, \bar{w}_{e}\right\}$. However, the number of extreme configurations is up to $2^{|E|}$ and it may be hard, in general case, to identify the configurations minimizing or maximizing $\delta_{f}(\boldsymbol{w})$. The computational complexity of deciding whether an element is possibly (necessarily) optimal depends on a particular problem $\mathcal{P}$. We provide some known and new results in this area in Section 3.2. The deviation interval for a given element can be efficiently computed if $\mathcal{P}$ is a matroidal problem, for instance $\mathcal{P}$ is Minimum Spanning Tree. Making use of the results obtained in $[21,27]$, it is easy to show that in this case $\underline{\delta}_{f}=\delta\left(\boldsymbol{w}_{\{f\}}^{-}\right)$and $\bar{\delta}_{f}=\delta\left(\boldsymbol{w}_{\{f\}}^{+}\right)$. However, this result is not valid for all problems $\mathcal{P}$.

If solution $X$ is not possibly optimal, then it is not optimal in configuration $\boldsymbol{w}_{X}^{-}$and $F\left(X, \boldsymbol{w}_{X}^{-}\right)>F^{*}\left(\boldsymbol{w}_{X}^{-}\right)$. If $F\left(Y, \boldsymbol{w}_{X}^{-}\right)=F^{*}\left(\boldsymbol{w}_{X}^{-}\right)$, then $F(X, \boldsymbol{w})>F(Y, \boldsymbol{w})$ for all configurations $\boldsymbol{w} \in \Gamma$. This implies $\bar{\delta}_{X}>\bar{\delta}_{Y}$ and $X$ cannot be an optimal minmax regret solution. We have thus established that every optimal minmax regret solution $X$ is possibly optimal, which can be equivalently expressed as $\underline{\delta}_{X}=0$. Since every possibly optimal solution $X$ is composed of possibly optimal elements and every optimal solution under a worst case configuration for $X$ is composed of possibly optimal elements, the non-possibly optimal elements do not influence the computation of an optimal minmax regret solutions. In consequence, they can be removed from $E$. This general property has been proved for some particular problems in $[19,27]$.

We now show some relationships between the necessarily optimal elements and the optimal minmax regret solutions. The following auxiliary proposition is easy to prove:

Proposition 1. Let $X$ and $Y$ be two solutions such that $F\left(X, \boldsymbol{w}_{X}^{-}\right)=F\left(Y, \boldsymbol{w}_{X}^{-}\right)$. Then the following two statements are true:

(i) $F(X, \boldsymbol{w}) \geq F(Y, \boldsymbol{w})$ for all configurations $\boldsymbol{w} \in \Gamma$,

(ii) if $X$ is an optimal minmax regret solution, then $Y$ is also an optimal minmax regret solution.

Let $e \in E$ be a necessarily optimal element and let $X$ be an optimal minmax regret solution such that $e \notin X$. Solution $X$ must be possibly optimal, so it is optimal under configuration $\boldsymbol{w}_{X}^{-}$. Since $e$ is necessarily optimal, there must be an optimal solution $Y$ under $\boldsymbol{w}_{X}^{-}$such that $e \in Y$. In consequence, we get $F\left(Y, \boldsymbol{w}_{X}^{-}\right)=F\left(X, \boldsymbol{w}_{X}^{-}\right)$and Proposition 1 now implies that $Y$ is also an optimal minmax regret solution. We have thus established that any necessarily optimal element is a part of an optimal minmax regret solution. So, while constructing a minmax regret solution we can always add a single necessarily optimal element to it. Obviously, there may exist more necessarily optimal elements but, in general, we cannot add all of them to the constructed solution. We show that this obstacle only appears if there are some degenerate intervals associated with elements.

Observe first that if all weight intervals are nondegenerate, then for every two distinct solutions $X$ and $Y$ there exists a configuration $\boldsymbol{w}$ such that $F(X, \boldsymbol{w}) \neq F(Y, \boldsymbol{w})$. Indeed, 
if $F\left(X, \boldsymbol{w}_{E}^{-}\right) \neq F\left(Y, \boldsymbol{w}_{E}^{-}\right)$, then we are done. Otherwise, we can choose element $e \in Y \backslash$ $X$ and we get $F\left(X, \boldsymbol{w}_{\{e\}}^{+}\right)<F\left(Y, \boldsymbol{w}_{\{e\}}^{+}\right)$, which follows from the fact that interval $W_{e}$ is nondegenerate. The following theorem generalizes a result for Minimum Spanning Tree obtained in [27]:

Theorem 1. If all weight intervals are nondegenerate, then there exists an optimal minmax regret solution that contains all necessarily optimal elements.

Proof. We will show that under nondegenerate weight intervals there exists an optimal minmax regret solution, which is the unique optimal solution under some configuration $w \in \Gamma$. This immediately implies that this solution must contain all necessarily optimal elements.

If $X_{1}$ is an optimal minmax regret solution, then it is possibly optimal and it is optimal under configuration $\boldsymbol{w}_{X_{1}}^{-}$. If $X_{1}$ is a unique optimal solution under $\boldsymbol{w}_{X_{1}}^{-}$, then we are done. Otherwise, there is another solution $X_{2}$ that is optimal under $\boldsymbol{w}_{X_{1}}^{-}$, thus $F\left(X_{1}, \boldsymbol{w}_{X_{1}}^{-}\right)=$ $F\left(X_{2}, \boldsymbol{w}_{X_{1}}^{-}\right)$. Proposition 1 now implies $X_{2}$ is also an optimal minmax regret solution. Moreover, $F\left(X_{1}, \boldsymbol{w}\right) \geq F\left(X_{2}, \boldsymbol{w}\right)$ for all $\boldsymbol{w} \in \Gamma$. Again solution $X_{2}$ is possibly optimal and it must be optimal under $\boldsymbol{w}_{X_{2}}^{-}$. We can repeat this argument obtaining a sequence $X_{1}, X_{2}, \ldots, X_{k}$ of optimal minmax regret solutions such that $F\left(X_{1}, \boldsymbol{w}\right) \geq F\left(X_{2}, \boldsymbol{w}\right) \geq$ $\cdots \geq F\left(X_{k}, \boldsymbol{w}\right)$ for all $\boldsymbol{w} \in \Gamma$. No solution in this sequence can be repeated. Indeed, suppose $X_{i}=X_{j}$ for some $i \neq j$. From the construction of the sequence, it follows that $j \neq i+1$ and so $F\left(X_{i}, \boldsymbol{w}\right) \geq F\left(X_{i+1}, \boldsymbol{w}\right) \geq F\left(X_{j}, \boldsymbol{w}\right)=F\left(X_{i}, \boldsymbol{w}\right)$ for all configurations $\boldsymbol{w}$. Thus there exist two distinct solutions $X_{i}$ and $X_{i+1}$ that have the same weights under all configurations, which is impossible if all the weight intervals are nondegenerate. Since the number of feasible solutions is finite, the following two cases are possible: either we meet a solution $X$ that is the unique optimal one under $w_{X}^{-}$and we are done or we enumerate all feasible solutions. In the second case, let $X_{|\Phi|}$ be the last solution enumerated. Since all intervals are nondegenerate, there is a configuration $\boldsymbol{w}$ such that $F\left(X_{|\Phi|-1}, \boldsymbol{w}\right)>F\left(X_{|\Phi|}, \boldsymbol{w}\right)$. It holds $F\left(X_{1}, \boldsymbol{w}\right) \geq F\left(X_{2}, \boldsymbol{w}\right) \geq \cdots \geq F\left(X_{|\Phi|-1}, \boldsymbol{w}\right)>F\left(X_{|\Phi|}, \boldsymbol{w}\right) \geq F\left(X_{|\Phi|}, \boldsymbol{w}_{X_{|\Phi|}}\right)$ and $X_{|\Phi|}$ is the unique optimal solution under $\boldsymbol{w}_{X_{|\Phi|}^{-}}$.

\subsection{A characterization of optimality of elements}

This section is entirely devoted to the evaluation of optimality of elements. Let us denote by $\operatorname{Poss} \mathcal{P}(\operatorname{NeC} \mathcal{P})$ a decision problem in which one asks whether a given element $f \in E$ is possibly (necessarily) optimal in problem $\mathcal{P}$ with interval weights. Equivalently, we may ask whether $\underline{\delta}_{f}=0\left(\bar{\delta}_{f}=0\right)$. In the next sections we will discuss three basic problems $\mathcal{P}$.

\subsubsection{The shortest path problem}

In the deterministic Shortest Path problem the element set $E$ consists of all paths between two distinguished nodes $s$ and $t$ in a given directed or undirected graph $G=(V, E)$ and we wish to find a path of minimum total weight. We start by recalling the Poss LONGEST PATH problem: 


\section{Poss Longest Path}

Input: A connected acyclic digraph $G=(V, A), s \in V, t \in V$, weights on the arcs $a \in A$ are to be chosen from intervals $W_{a}=\left[\underline{w}_{a}, \bar{w}_{a}\right], \underline{w}_{a} \geq 0$, a specified arc $f \in A$.

Question: Is there a weight configuration $\boldsymbol{w} \in \Gamma$ for which arc $f$ belongs to a longest $s-t$ path in $G$ ?

The Poss Longest PATH problem arises in a criticality analysis in project scheduling, where uncertain task durations are modeled by intervals. This problem is strongly NPcomplete in general acyclic digraphs [8] and remains NP-complete even in planar digraphs of degree 3 [9]. Recently, Okada in [25] has studied Poss Shortest PATH in acyclic digraphs. In order to show its NP-completeness, he proposed a reduction from Poss LONGEST PATH. Unfortunately this reduction is incorrect. We now give a correct reduction.
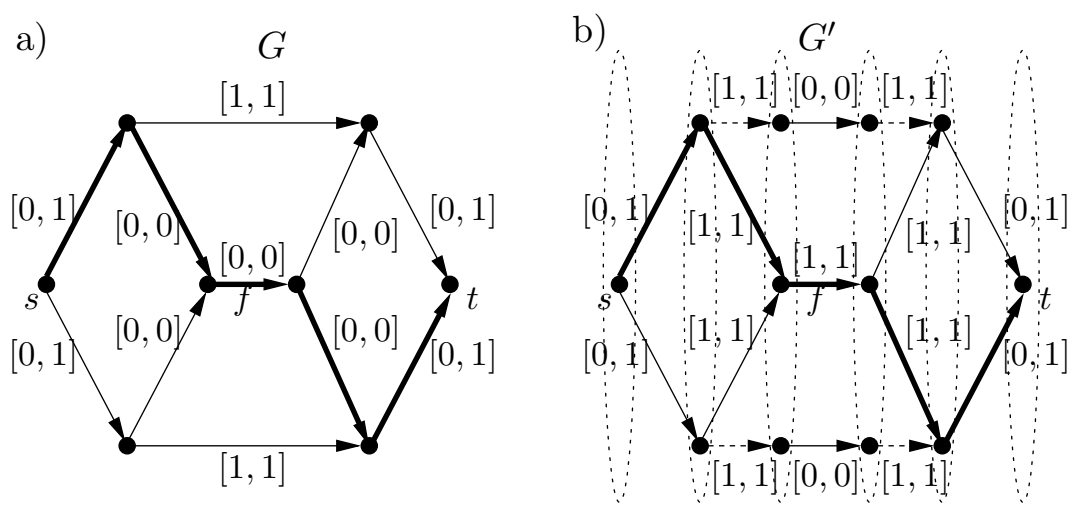

Figure 1: a) An instance of Poss Longest Path. b) The corresponding instance of Poss ShorTEST PATH.

Theorem 2. POss ShORTEST PATH is strongly NP-complete for acyclic digraphs and remains NP-complete for acyclic planar digraphs.

Proof. Let a digraph $G=(V, A)$ with interval arc weights and a distinguished arc $f$ be an instance of Poss Longest PAth. We construct an instance of Poss Shortest Path as follows. We first convert digraph $G$ into a layered digraph $G^{\prime}=\left(V^{\prime}, A^{\prime}\right)$ by adding to $G$ dummy nodes and dummy series arcs having weight intervals equal to [0,0] (in a layered digraph node set $V$ can be partitioned into disjoint subsets $V=\{s\} \cup V_{1} \cup \cdots \cup V_{k} \cup\{t\}$ and the arcs exist only from $s$ to $V_{1}$, from $V_{k}$ to $t$ and from $V_{i}$ to $V_{i+1}$ for $i=1, \ldots, k-1$ ). Observe that the dummy nodes only split some arcs of $G$ and there is one to one mapping between paths in $G$ and $G^{\prime}$. Nodes $s$ and $t$ and arc $f$ in graph $G^{\prime}$ are the same as in $G$. Since $G^{\prime}$ is layered, all paths between two specified nodes have the same number of arcs (see Figure 1b). We complete the reduction by changing the interval weights of the original arcs in following way $W_{a}^{\prime}=\left[M-\bar{w}_{a}, M-\underline{w}_{a}\right], a \in A^{\prime}$, where $M=\max \left\{\bar{w}_{a} \mid a \in A\right\}$. An example of an instance of POSS LONGEST PATH is shown in Figure 1a and the corresponding instance of Poss Shortest PATH is shown in Figure 1b. The dummy arcs are dashed. Now it is easily seen that arc $f$ belongs to a longest $s-t$ path in $G$ for some weight 
configuration if and only if it belongs to a shortest $s-t$ path in $G^{\prime}$ for some weight configuration. The Poss Shortest Path problem is in NP. It follows from the fact that an arc is possibly optimal if and only if it belongs to a possibly optimal path. A nondeterministic Turing machine first "guesses" a path $P$ such that $f \in P$. It can be then verified in polynomial time whether $P$ is possibly optimal (whether it is optimal in configuration $\left.\boldsymbol{w}_{P}^{-}\right)$.

From Theorem 2 we immediately get that computing $\underline{\delta}_{f}$ of a given element $f$ is strongly NP-hard for acyclic digraphs and remains NP-hard for acyclic planar digraphs. On the other hand, the problem of computing $\bar{\delta}_{f}$ of a given element $f$ in an acyclic digraph is polynomially solvable. The algorithms designed in [14] solve the problem of computing the upper bound on an arc float and they can be easily modified to compute the bound $\bar{\delta}_{f}$ in Shortest Path. Thus the NeC $\mathcal{P}$ problem in acyclic digraphs is polynomially solvable as well. However, the complexity status of this problem in general graphs is unknown.

Sometimes a special structure of the input graph allows us to compute efficiently the quantity $\underline{\delta}_{f}$. For instance, $\underline{\delta}_{f}$ (and $\bar{\delta}_{f}$ ) can be computed in $\mathcal{O}(|A|)$ time for any arc $f$ if graph $G$ has a series-parallel topology [10]. Also, in a general acyclic digraph $G$ it is possible to detect efficiently a subset of arcs, for which $\underline{\delta}_{f}>0[19,28]$.

\subsubsection{The assignment problem}

We now investigate the problem of evaluating the possible optimality of a specified element (edge) associated with Minimum Assignment. In this problem we are given a bipartite graph $G$ and $\Phi$ is the set of all perfect matchings in $G$.

Theorem 3. Poss Minimum Assignment is strongly NP-complete.

Proof. We show the strong NP-completeness of Poss Minimum Assignment by a reduction of Poss Shortest PATH, which is strongly NP-complete in acyclic digraphs (see Theorem 2). We claim that an instance of POss ShORTEST PATH is polynomially reducible to an instance of Poss Minimum Assignment.

Consider an instance of Poss Shortest Path. A digraph $G=(V, A)$ with interval arc weights $\left[\underline{w}_{i j}, \bar{w}_{i j}\right],(i, j) \in A$, being a part of this instance, is acyclic so the set $V=$ $\{1,2, \ldots, n\}$ can be labeled in such a way that $i<j$ for each $\operatorname{arc}(i, j) \in A, s=1$ and $t=n$. We assume that $f=(p, q), p, q \in V$, is the specified arc whose possible optimality is to be evaluated. We now use the transformation constructed by Hoffman and Markovitz [15]. We create a bipartite graph $G^{\prime}=\left(V_{1}^{\prime} \cup V_{2}^{\prime}, E^{\prime}\right)$, where $V_{1}^{\prime}=\{1, \ldots, n-1\}, V_{2}^{\prime}=\left\{2^{\prime}, \ldots, n^{\prime}\right\}$. If $(i, j) \in A$, then edge $\left\{i, j^{\prime}\right\}$ with weight interval $\left[\underline{w}_{i j}, \bar{w}_{i j}\right]$ is added to $E^{\prime}$. We also add to $E^{\prime}$ the edges of the form $\left\{i, i^{\prime}\right\}$ for $i=2, \ldots n-1$ with weight intervals $[0,0]$. We complete the construction by setting edge $f=\left\{p, q^{\prime}\right\}$ whose possible optimality is to be evaluated. The construction of $G^{\prime}$ is done in a time bounded by polynomial in the size of Poss SHORTEST PATH. A sample reduction is shown in Figure 2.

It can be verified [15] that there is one to one mapping from paths in $G$ to assignments in $G^{\prime}$. Let $\pi=\left(j_{0}, j_{1}, \ldots, j_{k}\right), j_{0}=1$ and $j_{k}=n$ be a sequence of nodes that forms a path 

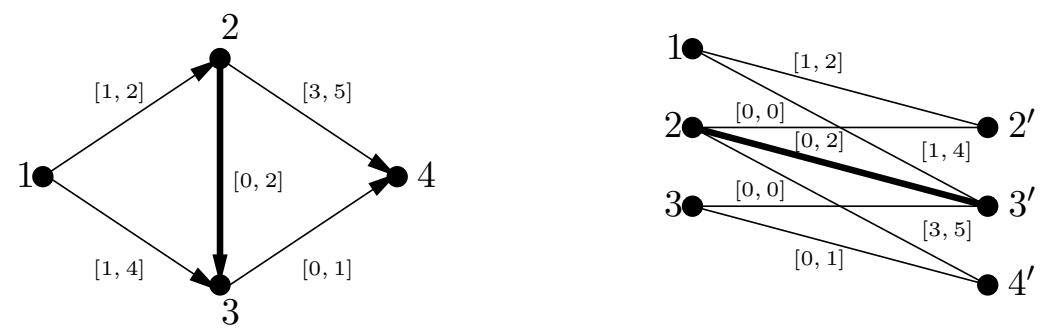

Figure 2: An instance of Poss Shortest Path and the corresponding instance of Poss Minimum Assignment.

in $G$. The corresponding assignment $\mathcal{A}$ is constructed as follows: node $j_{i}$ is paired with $j_{i+1}^{\prime}$ for $i=0, \ldots, k-1$; every node $j \notin \pi$ is paired with $j^{\prime}$. On the other hand, let $\mathcal{A} \subset E$ be an assignment in $G^{\prime}$. It must contain the subset of edges $\left\{\left\{j_{0}, j_{1}^{\prime}\right\},\left\{j_{1}, j_{2}^{\prime}\right\}, \ldots,\left\{j_{k-1}, j_{k}^{\prime}\right\}\right\}$, where $j_{0}<j_{1}<\cdots<j_{k-1}<n, j_{0}=1$ and $j_{k}=n$. Moreover, it is not difficult to verify that if $j \notin\left\{j_{0}, \ldots, j_{k-1}\right\}$ and $j \neq n$, then edge $\left\{j, j^{\prime}\right\}$ must belong to $\mathcal{A}$. Observe that the sequence of nodes $\left(j_{0}, j_{1}, \ldots, j_{k}\right)$ is a path from 1 to $n$ in $G$.

From the construction of $G^{\prime}$, it follows that one can use $\boldsymbol{w}$ to denote the same configuration of weights both in $G$ and $G^{\prime}$. It follows from the fact that all the additional edges in $G^{\prime}$ have degenerate weight intervals $[0,0]$. For any configuration $\boldsymbol{w}$ each path $\pi$ from 1 to $n$ in $G$ has a corresponding assignment $\mathcal{A}$ of the same weight in $G^{\prime}$ and the converse is also true. Consequently, an optimal path $\pi^{*}$ in $\boldsymbol{w}$ gives an optimal assignment $\mathcal{A}^{*}$ in $\boldsymbol{w}$ and vice versa. Moreover, $(p, q) \in \pi^{*}$ if and only if $\left\{p, q^{\prime}\right\} \in \mathcal{A}^{*}$. Thus arc $g=(p, q)$ is possibly optimal in Shortest PATH if and only if $f=\left\{p, q^{\prime}\right\}$ is possibly optimal in the corresponding Minimum Assignment. The Poss Minimum Assignment problem is in $\mathrm{NP}$ and the proof is similar to the proof in Theorem 2.

We can thus see that computing the value of $\underline{\delta}_{f}$ in Minimum Assignment is strongly NP-hard. However, the complexity status of the problems of computing $\bar{\delta}_{f}$ and checking the necessary optimality of edge $f$ is unknown.

\subsubsection{The minimum s-t cut problem}

Consider the problem of evaluating the optimality of a specified element (arc) $f$ associated with Minimum S-T CUT. In this problem $\Phi$ consists of all $s-t$ cuts in a given directed graph $G$. If $V_{1} \cup V_{2}$ is a partition of the node set $V$ such that $s \in V_{1}$ and $t \in V_{2}$, then a cut is formed by all arcs that start in $V_{1}$ and end in $V_{2}$. We will show that Poss Minimum S-T CUT is computationally intractable even if an input graph is planar. Recall that a graph is planar if it can be embedded in the plane without crossing arcs. An embedding of a planar digraph partitions the plane into separate regions called faces. Exactly one such face is unbounded and it is called the outer face. A right face of an arc $a=(i, j) \in A$ is the face which is on the right hand side of $(i, j)$ when traversing this arc from $i$ to $j$. Similarly we define the left face of $a$. 
Suppose that $G=(V, A)$ is a directed planar graph with two distinguished nodes $s$ and $t$. We assume, without loss of generality, that no $\operatorname{arc}$ enters $s$ and no arc leaves $t$. Such arcs can be removed from $G$ because they cannot be a part of any cut in $G$. We also assume that $G$ is given as a plane representation, in which $s$ and $t$ touch the outer face. The digraph $G$ is associated with a digraph $G^{*}=\left(V^{*}, A^{*}\right)$ called a dual digraph. The dual digraph is constructed as follows. We first modify $G$ by introducing an artificial arc $(t, s)$ in the outer face of $G$, so that this arc is directed clockwise when looking from the inside of $G$. The nodes of $G^{*}$ are the faces of the modified digraph $G$. For every arc $a \in A$, except for the artificial one, we add to $A^{*}$ arc $a^{*}$ that intersects $a$ and joins the nodes in the faces on either side of it; arc $a^{*}=\left(i^{*}, j^{*}\right)$ is oriented in such a way that node $i^{*}$ is in the right face of $a=(i, j)$. Finally, $s^{*} \in V^{*}$ is the node corresponding to the face limited by the artificial arc $(t, s)$ in $G$ and $t^{*} \in V^{*}$ is the node that corresponds to the outer face of $G$. It is easy to verify that $G^{*}$ is also a planar digraph and the dual of $G^{*}$ is $G$. An example of a planar digraph and its dual are shown in Figure 3. The construction of the dual graph $G^{*}$ can be done in polynomial time (see, e.g. $[1,23]$ ).

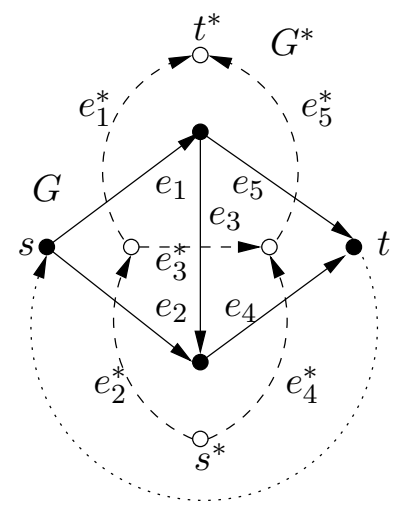

Figure 3: Construction of a dual graph $G^{*}$ (dashed arcs).

Let $V_{1} \cup V_{2}$ be a partition of $V$ such that $s \in V_{1}$ and $t \in V_{2}$. A cut $C$, that corresponds to this partition, is said to be uniformly directed $s-t$ cut if no $\operatorname{arcs}$ in $G$ lead from $V_{2}$ to $V_{1}$. The following theorem characterizes a planar digraph and its dual:

Theorem 4 ([1, 23]). Let $G$ be a planar acyclic digraph and let $G^{*}$ be its dual. Then $P=\left\{a_{1}, a_{2}, \ldots, a_{k}\right\}$ is a simple path from $s$ to $t$ in $G$ if and only if $C=\left\{a_{1}^{*}, a_{2}^{*}, \ldots, a_{k}^{*}\right\}$ is an uniformly directed $s^{*}-t^{*}$ cut in $G^{*}$

Hence there is one-to-one correspondence between simple paths in a directed planar graph and uniformly directed cuts in its dual. We will use this fact to prove the following result:

Theorem 5. Poss Minimum S-T Cut is NP-complete for planar digraphs.

Proof. We will construct a polynomial time reduction from Poss ShorTest PATH for acyclic planar digraphs, which is known to be NP-complete (see Theorem 2). Let an 
acyclic planar digraph $G=(V, A), s \in V, t \in V$, with interval arc weights $\left[\underline{w}_{a}, \bar{w}_{a}\right], a \in A$, and a specified arc $f=(k, l) \in A$ be an instance of Poss Shortest Path. We construct the corresponding instance of Poss Minimum S-T CUT as follows. We first construct a dual digraph $G^{*}=\left(V^{*}, A^{*}\right)$ for $G$. The interval weight of arc $a^{*} \in A^{*}$ is the same as the interval weight of the corresponding arc $a \in A$. Then for every $\operatorname{arc} a^{*}=\left(i^{*}, j^{*}\right) \in A^{*}$ we create the reverse $\operatorname{arc} b^{*}=\left(j^{*}, i^{*}\right)$ with interval weight $[M, M]$. This arc is called a dummy arc. We fix $M=|A| w_{\max }+1$, where $w_{\max }=\max _{a \in A} \bar{w}_{a}$. We denote by $G^{* *}$ the resulting digraph with dummy arcs. Finally, we distinguish $\operatorname{arc} f^{*}$ in $G^{* *}$. It is easily seen that digraph $G^{* *}$ is planar and it can be constructed from $G$ in polynomial time. A sample reduction is shown in Figure 4.

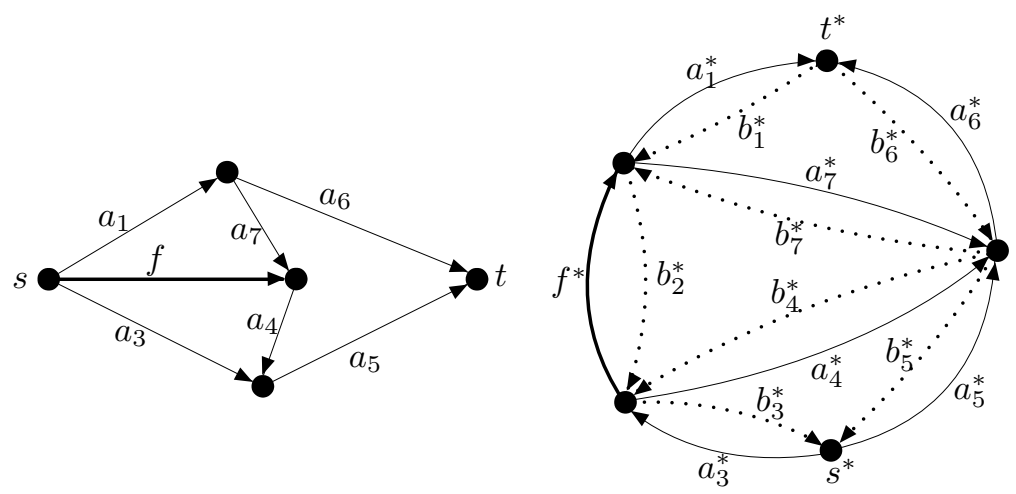

Figure 4: A sample reduction. Arcs $a_{i}^{*}$ have the same interval weights as $a_{i}$ and $\operatorname{arcs} b_{i}^{*}$ have interval weights $[M, M]$.

We claim that arc $f^{*}$ belongs to a minimum cut in $G^{* *}$ under some weight configuration if and only if $f$ belongs to a shortest path in $G$ under some weight configuration.

Observe first that every uniformly directed cut $C$ in $G^{*}$ is a cut in $G^{* *}$. It follows from the fact that adding dummy arcs to $G^{*}$ only backward arcs leading from $V_{2}$ to $V_{1}$ are created, where $V_{1} \cup V_{2}$ is a partition of nodes that corresponds to cut $C$. Consider a configuration $\boldsymbol{w}$ in $G^{* *}$. A minimum cut under $\boldsymbol{w}$ cannot use any dummy arc. Indeed, there is at least one cut in $G^{* *}$ that does not use any dummy arc. To see this consider a path from $s$ to $t$ in $G$. This path corresponds to a uniformly directed cut $C$ in $G^{*}$ and obviously $C$ is also the cut in $G^{* *}$. Every cut $C_{1}$ in $G^{* *}$, that uses a dummy arc, has the weight of at least $|A| w_{\max }+1$, which is strictly greater than the weight of $C$ under $\boldsymbol{w}$. Consequently, $C_{1}$ cannot be a minimum cut under $\boldsymbol{w}$. Now from Theorem 4 we get that path $P=\left\{a_{1}, a_{2}, \ldots, a_{k}\right\}$ is a shortest path in $G$ under some weight configuration if and only if cut $C=\left\{a_{1}^{*}, a_{2}^{*}, \ldots, a_{k}^{*}\right\}$ is a minimum cut in $G^{* *}$ under some weight configuration. Moreover, $f \in P$ if and only if $f^{*} \in C$. Poss Minimum s-T Cut is in NP and the reasoning is the same as for Poss ShorTest PATH in Theorem 2.

Similarly to Poss Minimum Assignment and Poss Shortest Path, computing $\underline{\delta}_{f}$ for a given arc $f$ in the considered problem is NP-hard. However, the problems of 
computing $\bar{\delta}_{f}$ and asserting whether $f$ is necessarily optimal remain open.

\section{Fuzzy combinatorial optimization problem}

In this section, we discuss a fuzzy version of problem $\mathcal{P}$, that is problem $\mathcal{P}$ with uncertain weights modeled by fuzzy intervals. We provide a possibilistic interpretation of the fuzzy problem together with some solution concepts, which can be viewed as a generalization of the minmax regret approach.

\subsection{Some basic notions of possibility theory}

A fuzzy set (see [11]) $\widetilde{A}$ is a reference set $\Omega$ together with mapping $\mu_{\widetilde{A}}$ from $\Omega$ into $[0,1]$, called a membership function. The value of $\mu_{\widetilde{A}}(v), v \in \Omega$, is interpreted as the degree of membership of $v$ in the fuzzy set $\widetilde{A}$. A $\lambda$-cut, $\lambda \in(0,1]$, of $\widetilde{A}$ is a classical set, i.e. $\widetilde{A}^{\lambda}=\left\{v \in \Omega: \mu_{\widetilde{A}}(v) \geq \lambda\right\}$. The cuts of $\widetilde{A}$ form a family of nested sets, i.e if $\lambda_{1} \geq \lambda_{2}$, then $\widetilde{A}^{\lambda_{1}} \subseteq \widetilde{A}^{\lambda_{2}}$. A fuzzy set in $\mathbb{R}$, whose membership function is normal, quasi-concave and upper semi-continuous is called a fuzzy interval (see [11]). A support of a fuzzy interval $\widetilde{A}$ is the set $\left\{v: \mu_{\widetilde{A}}(v)>0\right\}$ together with its closure and it will be denoted as $\widetilde{A}^{0}$. We will assume that the support of a fuzzy interval is bounded. It can be shown [11] that if $\widetilde{A}$ is a fuzzy interval with a bounded support, then $\widetilde{A}^{\lambda}$ is a closed interval for every $\lambda \in[0,1]$. We can thus represent a fuzzy interval $\widetilde{A}$ as a family of cuts $\widetilde{A}^{\lambda}=\left[\underline{a}^{\lambda}, \bar{a}^{\lambda}\right], \lambda \in[0,1]$. One can obtain the membership function $\mu_{\widetilde{A}}$ from the family of $\lambda$-cuts in the following way:

$$
\mu_{\widetilde{A}}(v)=\sup \left\{\lambda \in[0,1]: v \in \widetilde{A}^{\lambda}\right\}
$$

and $\mu_{\widetilde{A}}(v)=0$ if $v \notin \widetilde{A}^{0}$. Observe that a classical closed interval $A=[\underline{a}, \bar{a}]$ is a special case of a fuzzy one with membership function $\mu_{A}(v)=1$ if $v \in A$ and $\mu_{A}(v)=0$ otherwise. In this case we have $A^{\lambda}=[\underline{a}, \bar{a}]$ for all $\lambda \in[0,1]$. Another class of fuzzy intervals is formed by trapezoidal fuzzy intervals, denoted as $\left(\underline{a}, \bar{a}, \alpha_{A}, \beta_{A}\right), \alpha_{A}, \beta_{A}>0$. In this case we have $\tilde{A}^{\lambda}=\left[\underline{a}-(1-\lambda) \alpha_{A}, \bar{a}+(1-\lambda) \beta_{A}\right]$ for $\lambda \in[0,1]$.

Let us now recall a possibilistic interpretation of a fuzzy set. Possibility theory [11] is an approach to handle incomplete information with two dual measures: possibility and necessity, which are used to model available information. Both measures are built from a possibility distribution. Let a fuzzy set $\widetilde{A}$ be attached with a single-valued variable $\mathfrak{a}$. The membership function $\mu_{\widetilde{A}}$ is a possibility distribution, $\pi_{\mathfrak{a}}=\mu_{\widetilde{A}}$, which describes the set of more or less plausible, mutually exclusive values of the variable $\mathfrak{a}$. It is similar to a probability density. The value of $\pi_{\mathfrak{a}}(v)$ represents the possibility degree of the assignment $\mathfrak{a}=v$, i.e. $\Pi(\mathfrak{a}=v)=\pi_{\mathfrak{a}}(v)=\mu_{\widetilde{A}}(v)$. In particular, $\pi_{\mathfrak{a}}(v)=0$ means that $\mathfrak{a}=v$ is impossible and $\pi_{\mathfrak{a}}(v)>0$ means that $\mathfrak{a}=v$ is plausible. A degree of possibility can be viewed as an upper bound of a degree of probability. A detailed interpretation of the possibility distribution and some methods of obtaining it from the possessed knowledge are 
described in [11]. The possibility of an event "a $\in B$ ", denoted by $\Pi(\mathfrak{a} \in B)$, is as follows:

$$
\Pi(\mathfrak{a} \in B)=\sup _{v} \min \left\{\pi_{\mathfrak{a}}(v), \mu_{B}(v)\right\}
$$

where $B$ can be a fuzzy set. $\Pi(\mathfrak{a} \in B)$ evaluates the extent to which "a $\in B$ " is possibly true. If $B$ is a subset of $\mathbb{R}$, then $\mu_{B}$ is a characteristic function of $B$ and thus $\Pi(\mathfrak{a} \in B)=$ $\sup _{v \in B} \pi_{\mathfrak{a}}(v)$. The necessity of an event " $\mathfrak{a} \in B$ ", denoted by $\mathrm{N}(\mathfrak{a} \in B)$, is as follows:

$$
\mathrm{N}(\mathfrak{a} \in B)=1-\Pi(\mathfrak{a} \in \bar{B})=1-\sup _{v} \min \left\{\pi_{\mathfrak{a}}(v), 1-\mu_{B}(v)\right\}=\inf _{v} \max \left\{1-\pi_{\mathfrak{a}}(v), \mu_{B}(v)\right\},
$$

where $\bar{B}$ is the complement of $B$ and its membership function is $\mu_{\bar{B}}=1-\mu_{B} . \mathrm{N}(\mathfrak{a} \in B)$ evaluates the extent to which "a $\in B$ " is certainly true. If $B$ is a subset of $\mathbb{R}$, then $\mathrm{N}(\mathfrak{a} \in B)=\inf _{v \notin B}\left(1-\pi_{\mathfrak{a}}(v)\right)$.

\subsection{Fuzzy combinatorial optimization problem in the setting of possibility theory}

We now present a possibilistic formalization of problem $\mathcal{P}$, in which uncertainty of the element weights is modeled by fuzzy intervals $W_{e}, e \in E$. A membership function of $\widetilde{W}_{e}$ is regarded as a possibility distribution for the values of the unknown weight $\mathfrak{w}_{e}$, i.e. $\pi_{\mathfrak{w}_{e}}=\mu_{\widetilde{W}_{e}}, e \in E$. Thus the possibility degree of the assignment $\mathfrak{w}_{e}=v$ is $\Pi\left(\mathfrak{w}_{e}=\right.$ $v)=\pi_{\mathfrak{w}_{e}}(v)=\mu_{\widetilde{W}_{e}}(v)$. Let $\boldsymbol{w}=\left(v_{e}\right)_{e \in E}$ be a configuration of the element weights. The configuration $\boldsymbol{w}$ represents a state of the world in which $\mathfrak{w}_{e}=v_{e}$, for all $e \in E$. It defines an instance of problem $\mathcal{P}$ with the deterministic weights $\left(v_{e}\right)_{e \in E}$. Assuming that weights are unrelated to one another, the degree of possibility of a configuration $\boldsymbol{w}=\left(v_{e}\right)_{e \in E}$ is obtained by the following joint possibility distribution on configurations induced by $\widetilde{W}_{e}$, $e \in E($ see $[10,12])$ :

$$
\pi(\boldsymbol{w})=\Pi\left(\wedge_{e \in E}\left(\mathfrak{w}_{e}=v_{e}\right)\right)=\min _{e \in E} \Pi\left(\mathfrak{w}_{e}=v_{e}\right)=\min _{e \in E} \mu_{\widetilde{W}_{e}}\left(v_{e}\right) .
$$

Let us denote by $\mathcal{P}^{\lambda}, \lambda \in[0,1]$, the interval-valued problem $\mathcal{P}$ with element weights $\tilde{W}_{e}^{\lambda}=$ $\left[\underline{w}_{e}^{\lambda}, \bar{w}_{e}^{\lambda}\right], e \in E$. Note that the configuration set in $\mathcal{P}^{\lambda}$ is composed of all configurations $\boldsymbol{w}$ such that $\pi(\boldsymbol{w}) \geq \lambda$, that is whose possibility of occurrence is not less than $\lambda$. In the next sections we will show that the optimality evaluation and the problem of choosing a solution under fuzzy weights can be reduced to examining a family of interval problems $\mathcal{P}^{\lambda}, \lambda \in[0,1]$. In consequence, the results obtained for the interval-valued case can be applied to the fuzzy problems as well.

\subsubsection{The optimality evaluation and fuzzy deviation interval}

According to possibility theory, the degrees of possibility and necessity that a solution $X \in \Phi$ is optimal are defined as follows:

$$
\begin{aligned}
& \Pi(X \text { is optimal })=\sup _{\{\boldsymbol{w}: X \text { is optimal to } \mathcal{P} \text { for } \boldsymbol{w}\}} \pi(\boldsymbol{w}), \\
& \mathrm{N}(X \text { is optimal })=1-\Pi(X \text { is not optimal })=\inf _{\{\boldsymbol{w}: X \text { is not optimal to } \mathcal{P} \text { for } \boldsymbol{w}\}}(1-\pi(\boldsymbol{w})) \cdot(7)
\end{aligned}
$$


Similarly, the degrees of possibility and necessity that an element $f \in E$ is optimal are defined as follows:

$$
\begin{aligned}
& \Pi(f \text { is optimal })=\sup _{\{\boldsymbol{w}: f \text { is optimal to } \mathcal{P} \text { for } \boldsymbol{w}\}} \pi(\boldsymbol{w}), \\
& \mathrm{N}(f \text { is optimal })=1-\Pi(f \text { is not optimal })=\inf _{\{\boldsymbol{w}: f \text { is not optimal to } \mathcal{P} \text { for } \boldsymbol{w}\}}(1-\pi(\boldsymbol{w})) .
\end{aligned}
$$

It is easy to check that $\Pi(X$ is optimal $) \leq \min _{e \in X} \Pi(e$ is optimal $)$ and $\mathrm{N}(X$ is optimal $) \leq$ $\min _{e \in X} \mathrm{~N}(e$ is optimal $)$.

We now show that, similarly to the interval-valued case (see Section 3), we can express the degrees of possible and necessary optimality in terms of a deviation interval. In the fuzzy case, however, the deviation interval becomes a fuzzy one and it represents a possibility distribution for a solution (element) deviation. The possibility distribution that represents more or less plausible values of deviation $\delta_{X}$ of a solution $X$ is defined in the following way:

$$
\Pi\left(\delta_{X}=v\right)=\mu_{\tilde{\Delta}_{X}}(v)=\sup _{\left\{\boldsymbol{w}: \delta_{X}(\boldsymbol{w})=v\right\}} \pi(\boldsymbol{w}),
$$

where $\Pi\left(\delta_{X}=v\right)$ stands for the possibility degree that $\delta_{X}=v$. Since the statement " $X$ is optimal under $\boldsymbol{w}$ " is equivalent to the condition $\delta_{X}(\boldsymbol{w})=0$, we get the following relationships between optimality degrees (6), (7) and deviation (10):

$$
\begin{aligned}
& \Pi(X \text { is optimal })=\sup _{\left\{\boldsymbol{w}: \delta_{X}(\boldsymbol{w})=0\right\}} \pi(\boldsymbol{w})=\Pi\left(\delta_{X}=0\right)=\mu_{\tilde{\Delta}_{X}}(0), \\
& \mathrm{N}(X \text { is optimal })=1-\sup _{\left\{\boldsymbol{w}: \delta_{X}(\boldsymbol{w})>0\right\}} \pi(\boldsymbol{w})=\mathrm{N}\left(\delta_{X}=0\right)=1-\sup _{v>0} \mu_{\tilde{\Delta}_{X}}(v) .
\end{aligned}
$$

Using (3) we can express $\mu_{\tilde{\Delta}_{X}}$ in the following way:

$$
\mu_{\tilde{\Delta}_{X}}(v)=\sup \left\{\lambda: v \in \tilde{\Delta}_{X}^{\lambda}\right\}
$$

where $\tilde{\Delta}_{X}^{\lambda}=\left[\underline{\delta}_{X}^{\lambda}, \bar{\delta}_{X}^{\lambda}\right], \lambda \in[0,1]$, is the interval of possible values of deviation of solution $X$ in problem $\mathcal{P}^{\lambda}$. It is easy to verify that $\underline{\delta}_{X}^{\lambda}$ is a nondecreasing and $\bar{\delta}_{X}^{\lambda}$ is a nonincreasing function of $\lambda$. We thus have

$$
\Pi(X \text { is optimal })=\mu_{\tilde{\Delta}_{X}}(0)=\sup \left\{\lambda: 0 \in \Delta_{X}^{\lambda}\right\}=\sup \left\{\lambda: \underline{\delta}_{X}^{\lambda}=0\right\}
$$

and $\Pi(X$ is optimal $)=0$ if $\underline{\delta}_{X}^{0}>0$. A similar reasoning leads to the following equality:

$$
\mathrm{N}(X \text { is optimal })=1-\inf \left\{\lambda: \bar{\delta}_{X}^{\lambda}=0\right\}
$$

and $\mathrm{N}(X$ is optimal $)=0$ if $\bar{\delta}_{X}^{1}>0$. Exactly the same reasoning can be applied to elements. It is enough to replace $X$ with $f$ in formulae (10)-(13).

Formulae (12) and (13) suggest a method for computing the optimality degrees. In order to compute the degree of possible optimality we need to find the largest value of 
$\lambda \in[0,1]$ for which $\underline{\delta}_{X}^{\lambda}=0$ (resp. $\underline{\delta}_{f}^{\lambda}=0$ ), which is equivalent to asserting whether $X$ (resp. $f$ ) is possibly optimal in $\mathcal{P}^{\lambda}$. This can be done by halving the $[0,1]$ interval (a binary search) and solving a sequence of the interval-valued problems $\mathcal{P}^{\lambda}$. Computing the degree of necessary optimality is similar. Hence both degrees for a given solution or element can be computed in $\mathcal{O}\left(f(n) \log \epsilon^{-1}\right)$ time, where $\epsilon>0$ is a given precision and $f(n)$ is the time required to solve the corresponding interval problem $\mathcal{P}^{\lambda}$. We can see now that the running time heavily relies on the interval case because it boils down to optimality analysis on $\lambda$-cuts. In consequence, if we can characterize efficiently the optimality of a solution (element) in the interval case, then we can compute efficiently its degrees of optimality as well. Recall, that deciding whether an element is possibly optimal for some basic problems is NP-hard. So, computing the degree of possible optimality in this case is also NP-hard. It follows from the fact that the classical closed interval is a special case of a fuzzy one.

For a given solution $X \in \Phi$ it is sometimes possible to determine the whole possibility distribution $\mu_{\tilde{\Delta}_{X}}$. Making use of the properties shown in Section 3, we can compute the bounds $\underline{\delta}_{X}^{\lambda}$ and $\bar{\delta}_{X}^{\lambda}$ of $\tilde{\Delta}_{X}^{\lambda}$. That is, we compute the deviations of $X$ in two extreme configurations $\boldsymbol{w}_{X}^{-\lambda}$ and $\boldsymbol{w}_{X}^{+\lambda}$. In $\boldsymbol{w}_{X}^{-\lambda}$ we fix the weights of elements $e \in X$ to $\underline{w}_{e}^{\lambda}$ and the weights of the remaining elements to $\bar{w}_{e}^{\lambda}$. The configuration $\boldsymbol{w}_{X}^{+\lambda}$ is symmetric. It holds: $\underline{\delta}_{X}^{\lambda}=\sum_{e \in X} \underline{w}_{e}^{\lambda}-F^{*}\left(\boldsymbol{w}_{X}^{-\lambda}\right), \lambda \in[0,1]$ and $\bar{\delta}_{X}^{\lambda}=\sum_{e \in X} \bar{w}_{e}^{\lambda}-F^{*}\left(\boldsymbol{w}_{X}^{+\lambda}\right), \lambda \in[0,1]$. In order to compute functions $F^{*}\left(\boldsymbol{w}_{X}^{-\lambda}\right)$ and $F^{*}\left(\boldsymbol{w}_{X}^{+\lambda}\right)$ for $\lambda \in[0,1]$, some known parametric techniques can be applied. If $\underline{w}_{e}^{\lambda}$ and $\bar{w}_{e}^{\lambda}$ are linear functions of $\lambda$ for all $e \in E$, then for some particular problems such as Shortest Path or Minimum Spanning Tree their parametric counterparts can be efficiently solved (see, e.g. [13, 26]). In consequence, bounds $\underline{\delta}_{X}^{\lambda}$ and $\bar{\delta}_{X}^{\lambda}$ can be efficiently computed if trapezoidal fuzzy intervals are used to model the uncertain weights. Having a family of $\lambda$-cuts of $\tilde{\Delta}_{X}$ we can derive the possibility distribution $\mu_{\tilde{\Delta}_{X}}$ using (11). Contrary to solutions, computing $\mu_{\tilde{\Delta}_{f}}$ for a given element $f$ is more complex. It follows from the fact that there is no an easy method for determining extreme configurations that maximize and minimize element deviation in the interval case.

\subsubsection{Choosing a best solution}

We now present some concepts of choosing a solution in the fuzzy-valued problem $\mathcal{P}$. A best solution seems to be the one with the maximal degree of necessary optimality, i.e. an optimal solution to the following problem:

$$
\max _{X \in \Phi} \mathrm{N}(X \text { is optimal })=\max _{X \in \Phi} \mathrm{N}\left(\delta_{X}=0\right) .
$$

This solution is called a best necessarily optimal solution. For such solution with the highest degree we are certain that it is optimal. Using (13) we can rewrite problem (14) as follows:

$$
\begin{array}{ll}
\min & \lambda \\
\text { s.t. } & \bar{\delta}_{X}^{\lambda}=0, \\
& \lambda \in[0,1], \\
& X \in \Phi .
\end{array}
$$


If problem (15) is infeasible then $\mathrm{N}(X$ is optimal $)=0$ for all $X \in \Phi$. The constraint $\bar{\delta}_{X}^{\lambda}=0$ stands for the necessary optimality of $X$ in the interval-valued problem $\mathcal{P}^{\lambda}$. The problem (15) can be solved in polynomial time if problem $\mathcal{P}$ with deterministic weights is polynomially solvable. An algorithm is based on a binary search, that is, we halve the unit interval of possible values of $\lambda$ to compute the minimum value of $\lambda$ such that there exists a necessarily optimal solution in the interval-valued problem $\mathcal{P}^{\lambda}$. At each iteration finding a necessarily optimal solution, if it exists, can be done in polynomial, say in $f(n)$, time (see Section 3). Thus the overall complexity of the algorithm is $\mathcal{O}\left(f(n) \log \epsilon^{-1}\right)$, where $\epsilon>0$ is a given precision.

The criterion of choosing a solution used in (14) is very strong. Namely, a solution $X$ such that $\mathrm{N}(X$ is optimal $)>0$ may not exist or even if it exists, its necessary optimality degree may be very small. We apply now a more soft solution concept originally proposed for fuzzy linear programming in $[17,18]$. The idea consists in replacing the optimality requirement for a solution $\left(\delta_{X}=0\right)$ with a suboptimality one. Let us introduce a fuzzy goal $\widetilde{G}$ on the value of the deviation of $X$, where $\mu_{\widetilde{G}}:[0,+\infty) \rightarrow[0,1]$ is a nonincreasing function such that $\mu_{\widetilde{G}}(0)=1$. Function $\mu_{\widetilde{G}}$ is given in advance and the value of $\mu_{\widetilde{G}}\left(\delta_{X}\right)$ expresses the degree to which deviation $\delta_{X}$ satisfies a decision maker. The degree of necessity that a solution $X$ is soft optimal is defined as follows $[17,18]$ :

$$
\mathrm{N}(X \text { is soft optimal })=\inf _{\boldsymbol{w}} \max \left\{1-\pi(\boldsymbol{w}), \mu_{\widetilde{G}}\left(\delta_{X}(\boldsymbol{w})\right)\right\}
$$

Using (5) we can check that $\mathrm{N}(X$ is soft optimal $)=\mathrm{N}\left(\delta_{X} \in \widetilde{G}\right) . \mathrm{N}(X$ is soft optimal $)=\alpha$ means that for all configurations $\boldsymbol{w}$ such that $\pi(\boldsymbol{w})>1-\alpha$ it holds $\mu_{\widetilde{G}}\left(\delta_{X}(\boldsymbol{w})\right) \geq \alpha$ or equivalently $\delta_{X}(\boldsymbol{w}) \in \widetilde{G}^{\alpha}=\left[0, \mu_{\widetilde{G}}^{-1}(\alpha)\right]$, which represents the suboptimality of $X$. Function $\mu_{\widetilde{G}}^{-1}:[0,1] \rightarrow \mathbb{R} \cup\{+\infty\}$ is a pseudo-inverse of $\mu_{\widetilde{G}}$ that is $\mu_{\widetilde{G}}^{-1}(\alpha)=\sup \left\{v: \mu_{\widetilde{G}}(v) \geq \alpha\right\}$. Observe that if we define $\mu_{\widetilde{G}}(0)=1$ and $\mu_{\widetilde{G}}(x)=0$ for $x>0$, then $\mathrm{N}(X$ is soft optimal $)=$ $\mathrm{N}(X$ is optimal). Now, a more reasonable solution is an optimal one to the following problem:

$$
\max _{X \in \Phi} \mathrm{N}(X \text { is soft optimal }) .
$$

This solution is called a best necessarily soft optimal solution. It can be shown (the proof is similar to that in [18]) that problem (17) is equivalent to the following one:

$$
\begin{array}{ll}
\min & \lambda \\
\text { s.t. } & \bar{\delta}_{X}^{\lambda} \leq \mu_{\widetilde{G}}^{-1}(1-\lambda), \\
& \lambda \in[0,1], \\
& X \in \Phi .
\end{array}
$$

If problem (18) is infeasible then $\mathrm{N}(X$ is soft optimal $)=0$ for all solutions $X \in \Phi$. If $\lambda^{*}$ is the optimal objective value of (18) and $X$ is the best necessarily soft optimal solution, then $\mathrm{N}(X$ is soft optimal $)=1-\lambda^{*}$. Since $\bar{\delta}_{X}^{\lambda}$ is nonincreasing and $\mu_{\widetilde{G}}^{-1}(1-\lambda)$ is nondecreasing function of $\lambda$, problem (18) can also be solved by a binary search on $\lambda \in[0,1]$. We must 
find the smallest value of $\lambda$ for which condition $\bar{\delta}_{X}^{\lambda} \leq \mu_{\widetilde{G}}^{-1}(1-\lambda)$ is satisfied for some solution $X \in \Phi$. Since $\bar{\delta}_{X}^{\lambda}$ is the maximal regret of $X$ in $\mathcal{P}^{\lambda}$, a solution that minimizes $\bar{\delta}_{X}^{\lambda}$ is an optimal minmax regret solution in $\mathcal{P}^{\lambda}$. We thus can see that problem (18) consists of solving a family of minmax regret $\mathcal{P}^{\lambda}$ problems. Hence the concept of a necessary soft optimality is a natural extension of the minmax regret approach to the fuzzy case. Therefore, solving (18) is more complex than solving (15) and it depends on the complexity of the minmax regret version of problem $\mathcal{P}$.

\section{Conclusions}

In this paper, we have studied a general combinatorial optimization problem with ill-known element weights modeled by closed intervals and fuzzy intervals. Our aim was to present some general concepts and some relationships among them. We have discussed first the interval-valued case and then we have shown how the notions introduced for the intervalvalued problem can be naturally generalized to the fuzzy-valued one. We have also explored some computational aspects of the optimality evaluation. We have seen that characterizing the optimality of elements is, in general, more complex than characterizing the optimality of solutions.

The fuzzy problem has an interpretation in the setting of possibility theory. Applying this theory we can describe the notion of optimality and choose a robust solution under imprecision. The possibilistic analysis appears to be much easier than a probabilistic modeling. In particular, the computation of the possibility distribution of a solution or element deviation in a possibilistic framework is easier, than with a probabilistic model.

We have shown that the optimality evaluation and choosing a solution in the fuzzy problem is not harder than in the interval-valued case. In fact, every fuzzy problem boils down to solving a small number of interval problems. Thus the interval uncertainty representation seems to be a core problem in which the combinatorial structure of problem $\mathcal{P}$ plays a crucial role. Every result obtained for the interval problem can also be applied to its fuzzy counterpart as well. Furthermore, if some problem is hard in the interval case, then its fuzzy counterpart is not easier.

\section{Acknowledgements}

This work was partially supported by Polish Committee for Scientific Research, grant N N111 146433.

\section{References}

[1] V. Adlakha, B. Gladysz, J. Kamburowski, Minimum Flows in (s,t) Planar Networks, Networks 21 (1991) 767-773. 
[2] H. Aissi, C. Bazgan, D. Vanderpooten, Complexity of the min-max (regret) versions of cut problems, ISAAC 2005, LNCS 3827, pp. 789-798, 2005.

[3] H. Aissi, C. Bazgan, D. Vanderpooten, Complexity of the min-max and min-max regret assignment problems, Operations Research Letters 33 (2005) 634-640.

[4] H. Aissi, C. Bazgan, D. Vanderpooten, Min-max and min-max regret versions of combinatorial optimization problems: A survey, European Journal of Operational Research to appear 2009, doi:10.1016/j.ejor.2008.09.012.

[5] I. D. Aron, P. van Hentenryck, On the complexity of the robust spanning tree problem with interval data, Operations Research Letters 32 (2004) 36-40.

[6] I. Averbakh, V. Lebedev, Interval data minmax regret network optimization problems, Discrete Applied Mathematics 138 (2004) 289-301.

[7] I. Averbakh, V. Lebedev, On the complexity of minmax regret linear programming, European Journal of Operational Research 160 (2005) 227-231.

[8] S. Chanas, P. Zieliński, The computational complexity of the criticality problems in a network with interval activity times, European Journal of Operational Research 136 (2002) 541-550.

[9] S. Chanas, P. Zieliński, On the hardness of evaluating criticality of activities in a planar network with duration intervals, Operations Research Letters 31 (2003) 53-59.

[10] D. Dubois, H. Fargier, V. Galvagnon, On latest starting times and floats in activity networks with ill-known durations, European Journal of Operational Research 147 (2003) 266-280.

[11] D. Dubois, H. Prade, Possibility theory: an approach to computerized processing of uncertainty, Plenum Press, New York 1988.

[12] D. Dubois, H. Fargier, P. Fortemps, Fuzzy scheduling: modelling flexible constraints vs. coping with incomplete knowledge, European Journal of Operational Research 147 (2003) 231-252.

[13] D. Fernandez-Baca, G. Slutzki, D. Eppstein, Using sparsification for parametric minimum spanning tree problems, SWAT 1996, LNCS 1097, pp. 149-160, 1996.

[14] J. Fortin, P. Zieliński, D. Dubois, H. Fargier, Interval analysis in scheduling, CP 2005, LNCS 3709, pp. 226-240, 2005.

[15] A.J. Hoffman, H. Markowitz, A note on shortest path, assignment and transportation problems, Naval Research Logistics Quarterly 10 (1963) 375-379.

[16] M. Inuiguchi, Minimax regret solution to linear programming problems with an interval objective function, European Journal of Operational Research 86 (1995) 526-536. 
[17] M. Inuiguchi, On Possibilistic / Fuzzy Optimization, IFSA 200\%, LNAI 4527, pp. 351-360, 2007.

[18] M. Inuiguchi, M. Sakawa, Robust optimization under softness in a fuzzy linear programming problem, International Journal of Approximate Reasoning 18 (1998) 21-34.

[19] O. E. Karasan, M. C. Pinar, H. Yaman, The robust shortest path problem with interval data. Industrial Engineering Department, Bilkent University, 2001.

[20] A. Kasperski, P. Zieliński, An approximation algorithm for interval data minmax regret combinatorial optimization problems, Information Processing Letters 97 (2006) $177-180$.

[21] A. Kasperski, P. Zieliński, On combinatorial optimization problems on matroids with uncertain weights, European Journal of Operational Research 177 (2007) 851-864.

[22] P. Kouvelis, G. Yu, Robust Discrete Optimization and its applications. Kluwer Academic Publishers, Boston 1997.

[23] E. L. Lawler, Combinatorial Optimization: Networks and Matroids. Holt, Rinehart and Winston, New York 1976.

[24] R. Montemanni, L. M. Gambardella, A. V. Donati, A branch and bound algorithm for the robust shortest path problem with interval data, Operations Research Letters 32 (2004) 225-232.

[25] S. Okada, Fuzzy shortest path problems incorporating interactivity among paths, Fuzzy Sets and Systems 142 (2004) 335-357.

[26] N. Young, R. Tarjan, J. Orlin, Faster Parametric Shortest Path and Minimum Balance Algorithms, Networks 21 (1991) 205-221.

[27] H. Yaman, O. E. Karaşan, M. C. Pinar, The robust spanning tree problem with interval data, Operations Research Letters 29 (2001) 31-40.

[28] P. Zieliński, On computing the latest starting times and floats of activities in a network with imprecise durations, Fuzzy Sets and Systems 150 (2005) 53-76. 